on the surface, the great mass of the ocean consisting of cold water-water of $45^{\circ}, 40^{\circ}$, and of even a much lower temperature. At a little over half a mile of depth in the tropics the water has a temperature of $40^{\circ}$, and at the bottom it is still colder-ice-cold indeed. The ooze which is dredged from the bottom beneath the burning sun of the equator is so cold that the hand cannot be held in it for any time without great discomfort.

In the open ocean the temperature usually decreases with the depth, the coldest water being found at the bottom ; but sometimes there are limited areas where the temperature remains uniform for a mile or half a mile above the bottom. This has been shown to depend on the existence of barriers to free circulation, which exist on the floor of the ocean, and cause in a measure a resemblance to the conditions which are so marked in many partially enclosed seas, shut off by submarine barriers from general oceanic circulation, where the temperature is uniform, it may be, from a few fathoms below the surface to the bottom--for instance, in the Mediterranean and Seas of the Malayan Archipelago.

The low temperature of deep ocean water was acquired at the surface in high latitudes, chiefly in the high latitudes of the southern hemisphere. The salt warm water of the tropical regions, which is driven in relatively rapid currents along the eastern shores of South America, Africa, and Australia by the action of the prevailing winds, on reaching a southern latitude of $50^{\circ}$ or $55^{\circ}$ sinks on being cooled, and spreads over the floor of the ocean. A similar circulation takes place in the northern hemisphere, though modified in many ways by the peculiar configuration of the land : for instance, it is almost certain that the cold water at a temperature of $30^{\circ} \mathrm{F}$., which occupies the deeper part of the Norwegian Sea beyond the Wyville-Thomson Ridge, is the dense surface water of the Atlantic, which becomes cold and sinks as it passes northward in the extension of the Gulf Stream. Again, the relatively low temperature found on the eastern coasts of Africa and America seems largely due to the cold deep water which is drawn up to supply the place of the warm surface water driven forward by the trade winds.

While surface currents, both warm and cold, have at times considerable velocities, there is no evidence that rapid currents exist anywhere in the great deeps, on the contrary, the movements must be extremely slow and massive in character; the only exception seems to be on the crests of some ridges at moderate depths between volcanic islands or other similarly situated places.

Through the constant circulation in the ocean the gases of the atmosphere, which are everywhere absorbed at the surface of the sea according to the known laws of gas absorption, are borne down and thus enable myriads of living organisms to carry on their existence at all depths. The nitrogen remains at all times and places nearly constant, but frequently the proportion of oxygen is much reduced in deep water, owing to the processes of oxidation and respiration which are there going on.

The absorbed carbonic acid plays a most important and intricate roie in the economy of the ocean, owing to its tendency to reduce normal carbonate of lime and magnesia to solution in the form of bicarbonate; and to the rapid interchanges to which it is subject in consequence of vital processes. It probably receives large arditions from the bottom of the ocean, as an afterproduct of volcanic eruptions, and through the respiration of animals.

It is often supposed that hydrochemical actions go on with much greater activity in the deep sea where there may be a pressure of four or five tons on the square inch, but, while it would be convenient to assume it, there is no sufficient evidence that such is the case. The disintegrations, decompositions, and depositions which take place in the deposits are all similar to those which take place in shallow water or on land, and any chemical peculiarities occurring in inorganic or organic substances in great depths are probably due chiefly to the low temperature, almost perfect stillness, and the absence of light: for, although it may be admitted that some rays descend to much greater depths in the sea than is usually supposed, yet we must at present believe that none of them reach the greatest depths. The absorbed gases are probably but little affected by the great pressure of the superincumbent water, for in this connection it should be remembered that water is but little compressible; any substance which will sink to the bottom of a tumbler of water will in time sink to the bottom of the deepest ocean; this is true at least for all substances which are more compressible than water itself. The compressibility of water cannot, however, be neglected in oceanographical questions. In very great depths the lower layers are considerably compressed; for instance, in an ocean five miles deep, were the action of gravity suddenly to cease, the water would rise about 500 feet above its present level from expansion, a height sufficient to submerge nearly all the habitable land of the globe.

It remains to mention the investigations, which have recently been made, as to the change of level of the ocean, owing to the attraction of the masses of continental or other land-such, for instance, as that of the Himalayas for the water of the ocean to the south, by which the level of the Southern Indian Ocean is lowered some hundred feet; the bearing of this on the apparent elevation or submergence of land along coast-lines is evident, for the level of the sea, to which we refer all heights and depths, cannot be regarded as much more stable than the solid land itself.

(To be continued.)

\section{NEW PROCESS OF LIQUEFYING OXYGEN ${ }^{1}$}

I IQUID ethylene, the preparation and use of which I have already explained, shows, at its boiling-point under the pressure of the atmosphere, a temperature of at least $-103^{\circ} \mathrm{C}$., only some $10^{\circ}$ from the critical temperature of oxygen $\left(-\operatorname{II} 3^{\circ} \mathrm{C}\right.$.). It is understood how in the expansion of compressed and cooled

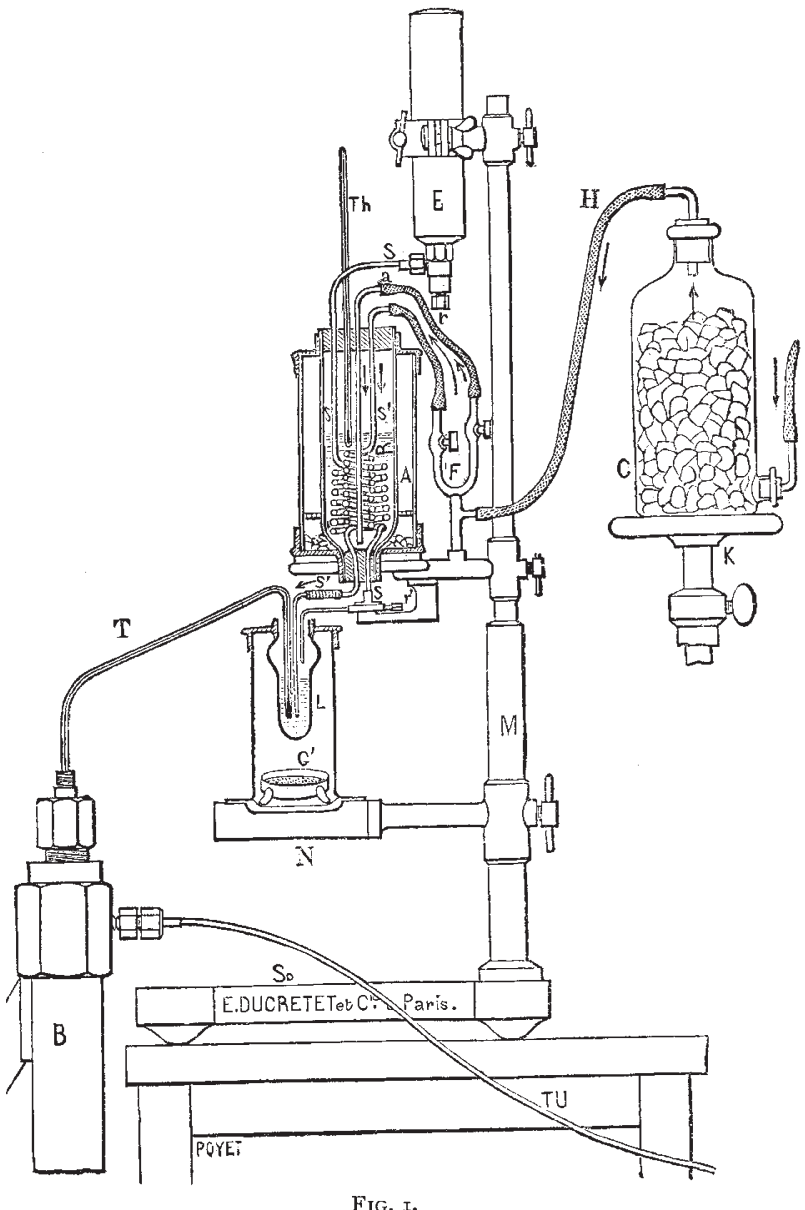

oxygen in the boiling ethylene the lowering of the temperature resulting from the expansion enabled me to establish "a tumultuous ebullition continuing an appreciable time." In

I From the Journal de Physique. By M. L. Cailletet. The illustrations have been kindly lent by MM. Ducretet et Cie, the manufacturers of M. Cailletet's apparatus. 
regulating the expansion so as to maintain a certain pressure in the tube, the oxygen is seen for some time completely liquefied.

When by means of the air-pump the evaporation of liquid ethylene is accelerated, as was done by Faraday with protoxide of nitrogen and carbonic acid, its temperature is reduced much below the critical point of oxygen.

With a view to avoiding the inconveniences and complications involved in the necessity of working in vacuo, I indicated liquid formène, which with the greatest ease achieves the liquefaction of oxygen and nitrogen. Notwithstanding these advantages, in consequence of the perfection to which I have recently brought the preparation and management of ethylene, it has seemed to me that this substance should be preferred to formene, and so, by means of boiling ethylene in open vessels, I have succeeded in obtaining a temperature sufficiently low for the complete liquefaction of oxygen.

The preparation of ethylene by means of sulphuric acid and alcohol is frequently impeded by the frothing of the material, terminating the experiment before the gas has been completely liberated. The admixture of sand, recommended by Wohler, does not always serve to counteract this frothing, but I have found the addition of a small quantity of vaseline efficacious in this respect.
The material I work with consists of 400 grammes of alcohol, 2000 grammes of sulphuric acid, and 15 to 20 grammes of vaseline. This is warmed in a glass globe, of 5 or 6 litres capacity, over a burner in the usual way. The gas is washed in two large flasks of caustic soda, and then collected in a water gasholder. By means of a mercury pump the ethylene is dried by passing through a flask of sulphuric acid and condensed in steel bottles having a screw tap.

Fig. I represents the apparatus I made use of to liquefy oxygen by the rapid evaporation of ethylene by means of a current of air or of refrigerated hydrogen. The liquid ethylene is inclosed in the bottle $\mathrm{E}$, which is fixed to a vertical support, with its mouth directed downwards, and is in communication with a copper worm, s s, of $3 \mathrm{~mm}$. to $4 \mathrm{~mm}$. in diameter, closed at its lower extremity by a screw cock, $r^{\prime}$. After the worm has been cooled to $-70^{\circ}$ by means of chloride of methylene in the manner I shall explain further on, the ethylene there accumulating possesses at this temperature but a weak tension, and it may therefore be run without sensible loss into the testtube, $L$, when the cock, $r^{\prime}$, is opened. This new arrangement I have adopted for ethylene and formene allows the liquefied gas to be cooled as well as though the whole reservoir containing it were of the same temperature as the worm.

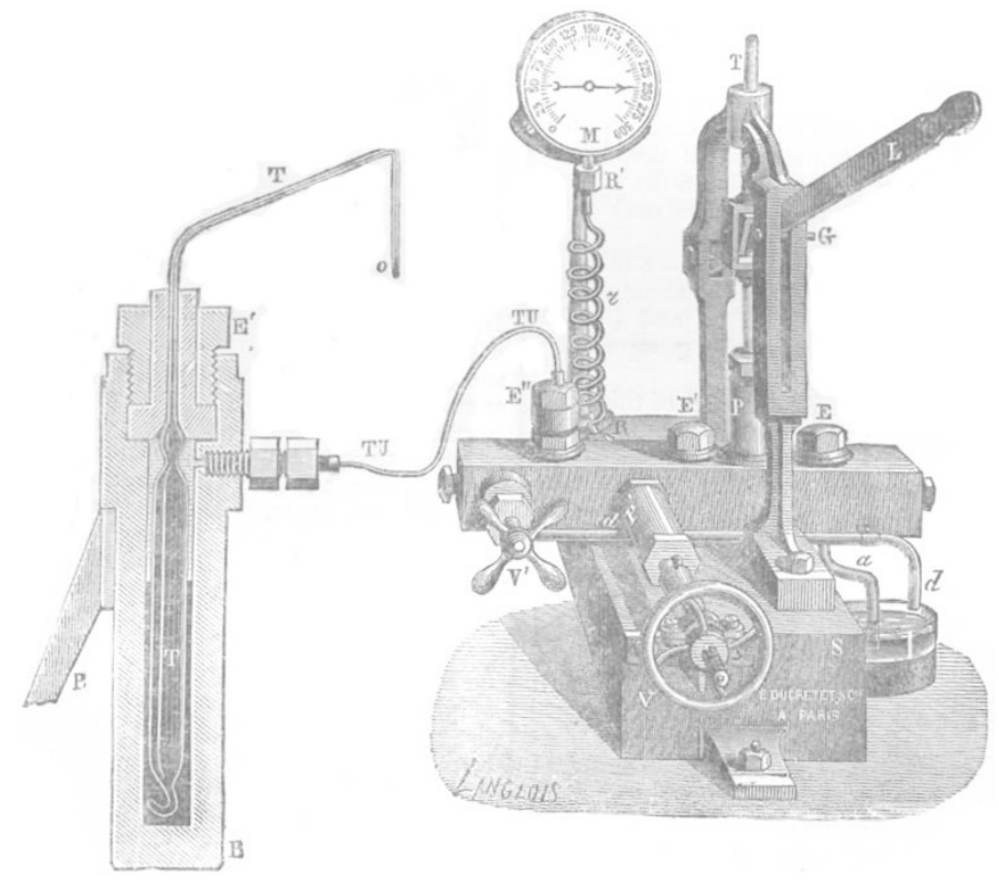

FIG. 2.

The glass test-tube $\mathrm{L}$ is arranged in a vessel containing, air dried by means of pumice and sulphuric $G^{\prime}$, and in this way hoar frost is prevented from being deposited on the refrigerated sides.

When the ethylene has been received in the test-tube $\mathrm{L}$, its evaporation is accelerated by passing through it a current of air, or, still better, of hydrogen dried by its passage in the vessel $\mathrm{C}$, containing chloride of calcium, and cooled in the worm $s^{\prime}$.

The two worms in which the air and the ethylene circulate are plunged into chloride of methylene which is rapidly evaporated by means of dry and cool air, and in this way a temperature of $-70^{\circ}$ is obtained.

Fig. 2 shows the arrangement of the oxygen apparatus and the compression pump. When the tube To is plunged into the ethylene, the evaporation of the latter is accelerated by gently opening the cock $F$, and blowing on to it the air or hydrogen cooled in the worm $\mathrm{s}^{\prime}$.

The pump is then brought into action, and the oxygen resolves into a colourless, transparent liquid, separated from the gas surmounting it by a perfectly sharp meniscus.

By means of a hydrogen thermometer, the construction of which I shall shortly explain, I have measured the temperature of the ethylene, which in one of my experiments was found to be $-123^{\circ} \mathrm{C}$. By dint of certain modifications effected in the apparatus I am in hopes of achieving a still lower temperature.

Altogether, I have proved that by quickening the evaporation of the ethylene by means of a current of air or hydrogen cooled to a low degree, its temperature is lowered much under that of the critical point of oxygen, and that in such a medium the oxygen liquefies most easilyr ${ }^{1}$

This experiment is so easy of accomplishment, that the practice of it may be commenced at once in laboratories, and be repeated in public lectures.

The apparatus I have described has been constructed with great care by $M$. Ducretet, and I have to thank M. Jamin for kindly permitting me to perform the experiments in the Physical Laboratory of the Sorbonne.

I M. E. Sainte-Claire Deville, engineer to the Gas Company of Paris, and son of my illustrious master, has now for some time, by my advice, been son of my illustrious master, has now for some time, by my advice, been studying the problem of lowering the temperature by means of the rapid evaporation of chloricle of methylene, and has established that, by sufficiently coling the injected air, temperatures varying from
be maintained nearly constant for several hours. 